\title{
TAME POLYHEDRA IN WILD CELLS AND SPHERES
}

\author{
R. B. SHER ${ }^{1}$
}

Abstract. It is shown that each arc on a disk $D$ in $E^{4}$ can be homeomorphically approximated by an arc in $D$ which is tame in $E^{4}$. Some applications of this are given. Also, we construct an everywhere wild $(n-1)$-sphere in $E^{n}, n \geqq 3$, each of whose arcs is tame in $E^{n}$.

1. Introduction. In [11] Seebeck showed that each arc on a disk $D^{2} \subset E^{n}, n \geqq 5$, can be homeomorphically approximated by arcs in $D^{2}$ which are tame in $E^{n}$. He also showed that if $n \geqq 4$ and $2 \leqq m \leqq n-2$, then there is an $m$-cell in $E^{n}$ which is wild at each of its points but each of whose arcs is tame in $E^{n}$.

In the current paper, we use some recent work of Bryant [3] to eliminate the missing case $n=4$ in the first result, and to sharpen the second result to the case $n \geqq 4$ and $2 \leqq m \leqq n-1$. We also give applications of the first result to problems in disk squeezing and cellularity.

We use $E^{n}$ to denote Euclidean $n$-space and $d$ to denote its usual metric. We sometimes regard $E^{n}$ as being the $\left(x_{n+1}=0\right)$-hyperplane in $E^{n+1}$. In this way, if $X \subset E^{n}$ is compact we may regard the suspension of $X$, denoted $\Sigma X$, as a subset of $E^{n+1}$. It is the join of $X$ with the set $\{(0, \cdots, 0,-1),(0, \cdots, 0,1)\} \subset E^{n+1}$.

If $M$ is a manifold we use Int $M$ and $\partial M$ for the interior and boundary of $M$ respectively. We use $\Delta^{n}$ to denote the standard $n$-simplex and $S^{n-1}$ to denote $\partial \Delta^{n}$.

2. Approximating 1-complexes by tame ones. Our main tool will be the following result due to Bryant [3, Corollary 2]. For completeness, we state it here. For convenience, we have modified its form slightly.

ThEOREM 1 (BRYANT). Let $A$ be an arc in $E^{n+1}, n \geqq 3$. Suppose there exists a dense subset $\left\{t_{1}, t_{2}, \cdots\right\}$ of $R$ such that $\left(E^{n} \times\left\{t_{i}\right\}\right)-A$ is 1-ULC for each $i$. Then $A$ is tame.

Received by the editors October 2, 1970.

AMS 1970 subject classifications. Primary 57A15, 57A45; Secondary 55A30, 57A60.

Key words and phrases. Everywhere wild, homeomorphically approximate, disk squeezing, cellularity.

${ }^{1}$ This research was supported in part by National Science Foundation Grant GP-19961. 
Having set forth the tool, we may now state the theorem whose proof occupies this section.

Theorem 2. Let $K$ be a finite 1-complex, $h: K \rightarrow \Delta^{2}$ an embedding, $f: \Delta^{2} \rightarrow E^{4}$ an embedding, and $\epsilon>0$. Then there is an embedding $g: K \rightarrow \Delta^{2}$ such that $f g: K \rightarrow E^{4}$ is tame and $d(f g(x), f h(x))<\epsilon$ for all $x \in K$. Furthermore, if $L$ is a subcomplex of $K$ such that $f h \mid L$ is tame, then $g$ can be chosen so that $g|L=h| L$.

Proof. The method of proof is inductive, so we may suppose that $K-L$ consists of a single 1 -simplex, say $\sigma$, with vertices $a$ and $b$. We shall construct an $\epsilon / 4$-push $k$ of $\left(E^{4}, f\left(\Delta^{2}\right)\right)$ and an embedding $g_{\sigma}: \sigma \rightarrow \Delta^{2}$ such that

(1) $g_{\sigma}|\{a, b\}=h|\{a, b\}$,

(2) $g_{\sigma}(\sigma) \cap h(K-\sigma)=\varnothing$,

(3) $d\left(k f g_{\sigma}(x), k f h(x)\right)<\epsilon / 2$ for all $x \in \sigma$, and

(4) $k f g_{\sigma}$ is tame.

Then $g$ is given by $g|K-\sigma=h| K-\sigma$ and $g \mid \sigma=g_{\sigma}$. By our assumption, $f g$ is tame on $L$ and, since $k$ is a push, $f g$ is tame on $\sigma$. Hence, $f g$ is tame by [4, Added in proof]. If $x \in \sigma$, then

$$
\begin{aligned}
& d(f g(x), f h(x)) \\
& \quad=d\left(k^{-1}\left[k f g_{\sigma}(x)\right], k^{-1}[k f h(x)]\right) \\
& \quad \leqq d\left(k^{-1}\left[k f g_{\sigma}(x)\right], k f g_{\sigma}(x)\right)+d\left(k f g_{\sigma}(x), k f h(x)\right)+d\left(k f h(x), k^{-1}[k f h(x)]\right) \\
& \quad<\epsilon / 4+\epsilon / 2+\epsilon / 4=\epsilon .
\end{aligned}
$$

To construct $k$ and $g_{\sigma}$ we begin by selecting a countable dense subset $\left\{t_{1}, t_{2}, t_{3}, \cdots\right\}$ of $R$. We may suppose that $\{f h(a), f h(b)\}$ $\subset E^{4}-\cup_{i=1}^{\infty} E^{3} \times\left\{t_{i}\right\}$. Let $Q_{i}$ be a subpolyhedron of $E^{4}$ triangulating $E^{3} \times\left\{t_{i}\right\}$, let $Q_{i}^{r}$ denote the $r$ th barycentric subdivision of $Q_{i}$ (with $\left.Q_{i}^{0}=Q_{i}\right)$, and let $T_{i}^{r}$ denote the 2-skeleton of $Q_{i}^{r}$. Let $\epsilon_{1}=\epsilon / 8$. By [11, Lemma 2], there is an $\epsilon_{1}$-push $k_{1}$ of $\left(E^{4}, f\left(\Delta^{2}\right) \cap T_{1}^{0}\right)$ such that $k_{1} f\left(\Delta^{2}\right)$ $\cap T_{1}^{0}$ is at most 0-dimensional. Let $D^{2}$ be a disk in $\Delta^{2}$ such that $h(\sigma)$ spans $D^{2}$ and $\partial D^{2} \cap h(K)=\{h(a), h(b)\}$. There is a proper embedding $g_{1}: \sigma \rightarrow D^{2}$ such that $g_{1}|\{a, b\}=h|\{a, b\}, k_{1} f g_{1}(\sigma) \cap T_{1}^{0}=\varnothing$, and $d\left(k_{1} f g_{1}(x), k_{1} f h(x)\right)<\epsilon_{1}$ for all $x \in \sigma$.

Inductively, suppose we have constructed, $\epsilon_{i}, k_{i}$, and $g_{i}$. Let

$$
\delta_{i}=\min \left\{d\left(k_{i} f g_{i}(\sigma), T_{1}^{i-1} \cup T_{2}^{i-2} \cup \cdots \cup T_{i}^{0}\right), \delta_{1}, \delta_{2}, \cdots, \delta_{i-1}\right\}
$$

and let

$$
\epsilon_{i+1}=\min \left\{\epsilon_{i} / 2, \delta_{i} / 2^{i+1}\right\} .
$$

By $\left[11\right.$, Lemma 2], there is an $\epsilon_{i+1}$-push $k_{i+1}^{\prime}$ of $\left(E^{4}, k_{i} f\left(\Delta^{2}\right) \cap\left(T_{1}^{i} \cup\right.\right.$ 
... $\left.\left.\cup T_{i}^{1} \cup T_{i+1}^{0}\right)\right)$ such that $k_{i+1}^{\prime} k_{i} f\left(\Delta^{2}\right) \cap\left(T_{1}^{i} \cup T_{2}^{i-1} \cup \ldots \cup T_{i}^{1} \cup\right.$ $\left.T_{i+1}^{0}\right)$ is at most 0-dimensional. Let $k_{i+1}=k_{i+1}^{\prime} k_{i}$ and let $g_{i+1}: \sigma \rightarrow D^{2}$ be a proper embedding such that

$$
\begin{gathered}
g_{i+1}|\{a, b\}=h|\{a, b\}, \\
k_{i+1} f g_{i+1}(\sigma) \cap\left(T_{1}^{i} \cup T_{2}^{i-1} \cup \ldots \cup T_{i}^{1} \cup T_{i+1}^{0}\right)=\varnothing,
\end{gathered}
$$

and

$$
d\left(k_{i+1} f g_{i+1}(x), k_{i+1} f g_{i}(x)\right)<\epsilon_{i+1} \text { for all } x \in \sigma .
$$

We may also pick $\epsilon_{i+1}$ small enough (depending on $k_{i}$ and $g_{i}$ ) so that $\left\{k_{i}\right\}_{i=1}^{\infty}$ converges to a push and $\left\{g_{i}\right\}_{i=1}^{\infty}$ converges to an embedding $[7]$.

Let $k=\lim _{i \rightarrow \infty} k_{i}$ and $g_{\sigma}=\lim _{i \rightarrow \infty} g_{i}$. Then $k$ is an $\epsilon / 4$-push of $\left(E^{4}\right.$, $f\left(\Delta^{2}\right)$ ). Property (1) holds since $g_{i}|\{a, b\}=h|\{a, b\}$ for all $i$, and property (2) holds since $g_{\sigma}$ embeds $\sigma$ in $D^{2}$. To see that property (3) holds, let $x \in \sigma$. Then

$$
\begin{aligned}
& d\left(k f g_{\sigma}(x), k f h(x)\right) \leqq d\left(k_{1} f g_{1}(x), k_{1} f h(x)\right) \\
& \quad+\sum_{i=1}^{\infty} d\left(k_{i} f g_{i}(x), k_{i+1} f g_{i}(x)\right)+\sum_{i=1}^{\infty} d\left(k_{i+1} f g_{i+1}(x), k_{i+1} f g_{i}(x)\right)<\epsilon_{1} \\
& \quad+\sum_{i=1}^{\infty} \epsilon_{i}+\sum_{i=1}^{\infty} \epsilon_{i+1}=2 \sum_{i=1}^{\infty} \epsilon_{i} \leqq \epsilon / 2 .
\end{aligned}
$$

We complete the proof by showing that $k f g_{\sigma}$ is tame. Let $x \in \sigma$ and $j, k$ be positive integers. Then

$$
\begin{aligned}
d\left(k f g_{\sigma}(x), T_{j}^{k}\right) \geqq & d\left(k_{j+k} f g_{j+k}(x), T_{j}^{k}\right) \\
& \quad-\sum_{i=j+k}^{\infty} d\left(k_{i} f g_{i}(x), k_{i+1} f g_{i+1}(x)\right) \geqq \delta_{j+k}-2 \sum_{i=j+k+1}^{\infty} \epsilon_{i} \\
\geqq & \delta_{j+k}-2 \sum_{i=j+k}^{\infty} \delta_{i} / 2^{i+1} \geqq \delta_{j+k}-2 \sum_{i=j+k}^{\infty} \delta_{j+k} / 2^{i+1} \\
= & \delta_{j+k}-2\left(\delta_{j+k} / 2^{j+k}\right)>0 .
\end{aligned}
$$

So $k f g_{\sigma}(\sigma) \cap\left[\bigcup_{j, k} T_{j}^{k}\right]=\varnothing$. Hence $\left(E^{3} \times\left\{t_{i}\right\}\right)-k f g_{\sigma}(\sigma)$ is 1-ULC for all $i$, and, by Theorem $1, k f g_{\sigma}$ is tame.

REMARKS. (i) The above proof is of course valid with $E^{4}$ replaced by $E^{n}, n \geqq 4$. However, if $n \geqq 5$, the approach of [11] is preferred.

(ii) The theorem is valid with $E^{4}$ replaced by $E^{n}, n \geqq 4$, and $\Delta^{2}$ replaced by a piecewise linear manifold $M^{m}, m \geqq 2$. This is due to the fact that any arc in $M$ can be approximated by one which lies on a 
disk. In addition, we need not assume that $M$ supports a piecewise linear structure since, by [8], some neighborhood of $h(K)$ in $M$ does.

3. Disk squeezing and cellularity. The theorem stated below was proved by R. J. Daverman and W. T. Eaton for the case $n=3$ [5] and $n \geqq 5$ [6]. Theorem 2 (in place of $[6$, Lemma A]) allows the proof of [6, Theorem 2] to go through in the case $n=4$.

The notation of the theorem is as follows: $\Delta_{2}$ is the disk in $E^{2}$ given by $\left\{(x, y) \mid x^{2}+y^{2} \leqq 1\right\}, \Delta_{1}$ is the arc spanning $\Delta_{2}$ and given by $\{(x, 0) \mid-1 \leqq x \leqq 1\}$, and $\pi$ is the projection of $\Delta_{2}$ onto $\Delta_{1}$ given by $\pi(x, y)=(x, 0)$.

Theorem 3 (Daverman and Eaton). Suppose $D^{2}$ is a disk in $E^{n}, n \geqq 3, g_{0}: \Delta_{2} \rightarrow D^{2}$ is a homeomorphism, $U$ is an open subset of $E^{n}$ with $D^{2}-g_{0}\left(\partial \Delta_{1}\right) \subset U$ and $\epsilon>0$. Then there exists a surjective map $f: E^{n} \rightarrow E^{n}$, a homeomorphism $g: \Delta_{2} \rightarrow D^{2}$, and a homeomorphism $h: \Delta_{1} \rightarrow$ $f\left(D^{2}\right)$ such that

(1) $f \mid E^{n}-D^{2} \rightarrow E^{n}-f\left(D^{2}\right)$ is a homeomorphism,

(2) $f \mid E^{n}-U=\mathrm{id}_{E^{n}-U}$,

(3) $f g=h \pi$,

(4) $d\left(g(x), g_{0}(x)\right)<\epsilon$ for all $x \in \Delta_{2}$, and

(5) $g\left|\partial \Delta_{1}=g_{0}\right| \partial \Delta_{1}$.

REMARK. (iii) It is interesting to note that, in case $n \neq 4$, the arcs $f^{-1}(p), p \in \operatorname{Int} f\left(D^{2}\right)$, are cellular in $E^{n}$ (definition below). This follows from [1, Corollary 5.5].

Applying Theorem 3 to a wedge of disks in $E^{n}$ we obtain the following result.

Corollary 4 (Daverman and EAton). Suppose that $P^{2}=\left(D_{1}^{2}, a\right)$ $\bigvee\left(D_{2}^{2}, a\right) \bigvee \cdots \vee\left(D_{k}^{2}, a\right) \subset E^{n}, n \geqq 3$, where $a \in \partial D_{i}^{2}$ if $i=1,2, \cdots, k$, and that $U$ is an open subset of $E^{n}$ with $P^{2} \subset U$. Then there exists $a$ surjective map $f: E^{n} \rightarrow E^{n}$, homeomorphisms $g_{i}: \Delta_{2} \rightarrow D_{i}^{2}$ and homeomorphisms $h_{i}: \Delta_{1} \rightarrow f\left(D_{i}^{2}\right)$ such that

(1) $f \mid E^{n}-P^{2} \rightarrow E^{n}-f\left(P^{2}\right)$ is a homeomorphism,

(2) $f \mid E^{n}-U=\operatorname{id}_{E^{n}-U}$,

(3) $f g_{i}=h_{i} \pi$ for $i=1,2, \cdots, k$, and

(4) $g_{i}((-1,0))=a=f(a)$ for $i=1,2, \cdots, k$.

Recall that a continuum $X$ in the interior of an $n$-manifold $M^{n}$ is cellular if there exist $n$-cells $C_{1}, C_{2}, \cdots$ in $M^{n}$ such that $C_{i+1} \subset \operatorname{Int} C_{i}$, $i=1,2, \cdots$, and $X=\bigcap_{i=1}^{\infty} C_{i}$.

For the construction of Example 6 we require the following lemma.

Lemma 5. If $X$ is a cellular continuum in $E^{n}$, then $\Sigma X$ is cellular in $E^{n+1}$. 
Proof. It suffices to show that if $U$ is an open set in $E^{n+1}$ with $\Sigma X \subset U$, then there is an $(n+1)$-cell $C$ such that $X \subset$ Int $C \subset C \subset U$. Since $X$ is cellular, there exists a locally flat $n$-cell $D$ in $E^{n}$ such that $X \subset$ Int $D$ and $\Sigma D \subset U$. We note that $\Sigma X$-(suspension points) lies in Int $(\Sigma D)$. Now, the $(n+1)$-cell $\Sigma D$ is locally flat since $D$ is locally flat [9]. Staying in $U$, we obtain $C$ from $\Sigma D$ by pasting a small $(n+1)$-cell to $\Sigma D$ at each suspension point.

We now construct some examples which give us an answer to the question raised in [12, Remark (viii)].

EXAMPLE 6. There exists a noncellular $k$-frame in $E^{n}, k \geqq 3, n \geqq 3$, each of whose $(k-1)$-frames is cellular.

Construction. For $n=3$ an explicit construction for such frames is given in $[12, \S 4]$. The frames are shown to be noncellular by showing that their complements fail to be simply connected.

Now suppose $K$ is a $k$-frame in $E^{n}$ with $\pi_{1}\left(E^{n}-K\right) \neq 0$ and each $(k-1)$-frame in $K$ cellular in $E^{n}$. Let $a$ denote the junction point of $K$. Since $\pi_{1}\left(E^{n}-K\right) \neq 0$, we find that $\pi_{1}\left(E^{n+1}-\Sigma K\right) \neq 0$. Hence $\Sigma K$ fails to be cellular. But, by Lemma 5 , if $L$ is a $(k-1)$-frame in $K$, then $\Sigma L$ is cellular in $E^{n+1}$.

We now squeeze $\Sigma K$ to obtain the desired $k$-frame in $E^{n+1}$ in two steps. First, we collapse the (tame) arc $\Sigma a$ to a point. This collapses $\Sigma K$ to a wedge of $k$ disks which we denote by $P^{2}$. While $P^{2}$ is not cellular, its proper sub-wedges are. Now apply Corollary 4 to squeeze $P^{2}$ to a $k$-frame. Again, the $k$-frame is not cellular, but its $(k-1)$ frames are.

REMARK. (iv) An easy proof of Theorem 2 (or its analog in $E^{n}$, $n \geqq 5$ ) can be given in the special case when $f$ is a suspension embedding; this would be applicable in the above construction. For this proof, we would simply note that each arc in a suspension disk can be approximated, modulo its endpoints by one whose interior is the union of a countable collection of "horizontal" and "vertical" arcs in the product structure of $E^{n}=E^{n-1} \times E^{1}$. Such arcs are known to be tame.

4. An example in codimension one. Seebeck shows [11] that if $n \geqq 4$ and $2 \leqq m \leqq n-2$, then there is an $m$-cell in $E^{n}$ which is wild at each of its points but each of whose arcs is tame. We show here how to use Theorem 1 to extend this result to the case $m=n-1$.

EXAMPLE 7. There exists an $(n-1)$-sphere in $E^{n}, n \geqq 3$, which is wild at each of its points, but each of whose arcs is tame.

Construction. If $n=3$, then Bing's "hooked rug" [2] provides our example. By suspending this example an appropriate number of times we obtain an $(n-1)$-sphere $S$ in $E^{n}$ which is wild at each point and 
which is the suspension of an $(n-2)$-sphere $S_{0}$ in $E^{n-1}$. Inductively, we may suppose that each arc in $S_{0}$ is tame in $E^{n-1}$. (That $S$ is wild at each point is shown in [10].) Let $A$ be an arc in $S$.

There is a dense subset $\left\{t_{1}, t_{2}, t_{3}, \cdots\right\}$ of $R$ such that $A \cap\left(E^{n-1}\right.$ $\left.\times\left\{t_{i}\right\}\right)$ is at most 0 -dimensional for each positive integer $i$. If $A \cap\left(E^{n-1} \times\left\{t_{i}\right\}\right) \neq \varnothing$, then $A \cap\left(E^{n-1} \times\left\{t_{i}\right\}\right)$ lies on the 2-sphere $S \cap\left(E^{n-1} \times\left\{t_{i}\right\}\right)$. (Unless, trivially, $t_{i}=-1$ or 1.) But the pair $E^{n-1} \times\left\{t_{i}\right\}, S \cap\left(E^{n-1} \times\left\{t_{i}\right\}\right)$ is homeomorphic to $\left(E^{n-1}, S_{0}\right)$. Hence, there is an arc $B \subset E^{n-1} \times\left\{t_{i}\right\}$ such that $A \cap\left(E^{n-1} \times\left\{t_{i}\right\}\right) \subset B$ and $B$ is tame in $E^{n-1} \times\left\{t_{i}\right\}$. But then $A \cap\left(E^{n-1} \times\left\{t_{i}\right\}\right)$ is tame in $E^{n-1} \times\left\{t_{i}\right\}$; hence, $E^{n-1} \times\left\{t_{i}\right\}-A \cap\left(E^{n-1} \times\left\{t_{i}\right\}\right)$ is 1-ULC. By Theorem $1, A$ is tame.

\section{REFERENCES}

1. Steve Armentrout, Homotopy properties of decomposition spaces, Trans. Amer. Math. Soc. 143 (1969), 499-507.

2. R. H. Bing, $A$ wild surface each of whose arcs is tame, Duke Math. J. 28 (1961), 1-15. MR 23 \#A630.

3. J. L. Bryant. On embeddings with locally nice cross-sections (to appear).

4. J. C. Cantrell, $n$-frames in Euclidean $k$-space, Proc. Amer. Math. Soc. 15 (1964), 574-578. MR 29 \#1627.

5. R. J. Daverman and W. T. Eaton, An equivalence for the embeddings of cells in a 3-manifold, Trans. Amer. Math. Soc. 145 (1969), 369-381. MR 40 \#3519.

6. - Each disk in $E^{n}$ can be squeezed to an arc (to appear).

7. Herman Gluck, Embeddings in the trivial range, Ann. of Math. (2) 81 (1965), 195-210. MR 30 \#3456.

8. John Hollingsworth and R. B. Sher, Triangulating neighborhoods in topological manifolds (to appear).

9. C. Lacher, Locally flat strings and half-strings, Proc. Amer. Math. Soc. 18 (1967), 299-304. MR 35 \#3670.

10. T. B. Rushing, Everywhere wild cells and spheres (to appear).

11. Charles L. Seebeck III, Tame arcs on wild cells (to appear).

12. R. B. Sher, Determining the cellularity of a 1-complex by properties of its arcs, Proc. Amer. Math. Soc. 26 (1970), 491-498.

University of Georgia, Athens, Georgia 30601 